\title{
Local Strain Distribution in Real Three-Dimensional Alveolar Geometries
}

\author{
S. M. K. Rausch, ${ }^{1}$ D. Haberthür,${ }^{2}$ M. Stampanoni, ${ }^{3,4}$ J. C. Schittny, ${ }^{2}$ and W. A. Wall ${ }^{1}$ \\ ${ }^{1}$ Institute for Computational Mechanics, Technische Universität München, Boltzmannstrasse 15, 85747 Garching, Germany; \\ ${ }^{2}$ Institute of Anatomy, University of Bern, Bern, Switzerland; ${ }^{3}$ Swiss Light Source, Paul Scherrer Institut, Villingen, Switzerland; \\ and ${ }^{4}$ Institute for Biomedical Engineering, University and ETH Zürich, Zurich, Switzerland
}

(Received 21 February 2011; accepted 12 May 2011; published online 24 May 2011)

Associate Editor Kerry Hourigan oversaw the review of this article.

\begin{abstract}
Mechanical ventilation is not only a life saving treatment but can also cause negative side effects. One of the main complications is inflammation caused by overstretching of the alveolar tissue. Previously, studies investigated either global strains or looked into which states lead to inflammatory reactions in cell cultures. However, the connection between the global deformation, of a tissue strip or the whole organ, and the strains reaching the single cells lining the alveolar walls is unknown and respective studies are still missing. The main reason for this is most likely the complex, sponge-like alveolar geometry, whose three-dimensional details have been unknown until recently. Utilizing synchrotron-based X-ray tomographic microscopy, we were able to generate real and detailed three-dimensional alveolar geometries on which we have performed finite-element simulations. This allowed us to determine, for the first time, a three-dimensional strain state within the alveolar wall. Briefly, precision-cut lung slices, prepared from isolated rat lungs, were scanned and segmented to provide a threedimensional geometry. This was then discretized using newly developed tetrahedral elements. The main conclusions of this study are that the local strain in the alveolar wall can reach a multiple of the value of the global strain, for our simulations up to four times as high and that thin structures obviously cause hotspots that are especially at risk of overstretching.
\end{abstract}

Keywords-Alveoli, Finite-element method, Local strains, Synchrotron-based X-ray tomographic microscopy.

\section{INTRODUCTION}

Acute Lung Injury (ALI) and Acute Respiratory Distress Syndrome (ARDS) are severe diseases with a high mortality rate. ${ }^{27}$ An initial release of inflammatory mediators triggers a diffuse inflammation of the lung parenchyma, leading to hypoxia and frequently to

Address correspondence to S. M. K. Rausch, and W. A. Wall, Institute for Computational Mechanics, Technische Universität München, Boltzmannstrasse 15, 85747 Garching, Germany. Electronic mail: rausch@lnm.mw.tum.de, wall@1nm.mw.tum.de multi-organ failure. It is known that ARDS and its lighter form ALI can be caused by either direct lung injury, like pneumonia or aspiration, or indirect lung injury, like sepsis or severe trauma. The introduction of protective ventilation protocols, including positive end-expiratory pressure (PEEP) and a decrease of tidal volume has led to a reduction in these mortality rates, but they still remain unsatisfactorily high. ${ }^{27}$ Using PEEP should prevent the lungs from partly collapsing, by not letting the pressure drop to zero at the end of expiration. The reduction of tidal volume should prevent the tissue from being overstretched during ventilation (volutrauma). Due to the fact that the lungs of ARDS patients are normally injured inhomogeneously, the air distributes unevenly throughout the lungs and the optimal level of PEEP, tidal volume etc. are hard to find for individual patients. This can lead to an overextension of the healthy parts, causing further complications, known as ventilator-induced lung injury (VILI). These complications include both mechanical damage of the tissue and activation of an inflammatory signaling cascade (biotrauma). How the ventilation exactly induces its deleterious effects is still unclear. Studies both in vitro and in vivo have found that both the pattern and the degree of stretch are important. ${ }^{6,9,10}$

A major problem is that there is no possibility to measure local deformations in vivo. For this reason a connection between the global deformation of a tissue strip or the whole organ and the strains reaching the single cells lining the alveolar walls is needed. However, due to the complex alveolar geometry the determination of this relationship is not straightforward.

Only a few papers addressed this topic so far. For instance, Wilson and Bachofen ${ }^{35}$ presented a twodimensional model for the relationship between surface and force bearing elements in the alveolar duct. This model allows an analysis of the relationship 
between the surface area, recoil pressure, lung volume, and surface tension. However, the alveolar wall thickness is neglected, i.e. there is no information about the deformation within the wall. A similar approach was used by Maksym et al. ${ }^{17}$ where they idealized individual septal walls in lung tissue strips as a two-dimensional network of elastin and collagen fibers arranged in parallel. As with the model of Wilson and Bachofen ${ }^{35}$ they only have one-dimensional wall deformation. Brewer et al. ${ }^{1}$ investigated immunofluorescently labeled alveolar walls in small tissue strips and followed their extension and changes in angle under uniaxial stretching, utilizing a fluorescence light microscopy. By comparing the microstrains, the relative length change in the wall segments, with the angle change they found a considerable heterogeneity. However, due to the imaging technique the authors were only able to investigate two-dimensional images of a three-dimensional deformation state. Furthermore, the alveolar walls were modeled as one-dimensional line elements, meaning bending effects were completely neglected. A similar method was used by Cavalcante et al. ${ }^{2}$ They investigated the influence of the tissue constituents in the alveolar wall. Among others, they compared the local extension of the alveolar wall with the global deformation by utilizing the same method as Brewer et al. ${ }^{1}$ DiRocco et al. ${ }^{8}$ investigated the dynamics of rat alveoli in vivo. They utilized videomicroscopy to image the alveoli located directly under the pleura. The change in area between inspiration and expiration of manually outlined alveoli was calculated for healthy and diseased lungs. The imaging technique is again two-dimensional and additional only subpleural alveoli can be investigated, which are pathologically not as important during ARDS. Perlman and Bhattacharya $^{20}$ utilized real-time confocal microscopy to determine the micromechanics of alveolar perimeter distension in perfused rat lungs. They were able to image a $2-\mu$ m-thick optical section $20 \mu \mathrm{m}$ under the pleura. Five to eight segments were identified within each alveolus, which is in contrast to the above mentioned authors who claim in their work that the alveoli always have six segments. The average length of these segments was compared for normal and hyper inflation. They found the segment distension to be heterogeneous within the single alveolus. Two shortcomings of this technique are that the alveolar extension is measured only in two dimensions and due to the imaging technique only alveoli located close to the pleura could be included. Another approach is to simulate the deformation of the alveolar wall utilizing the finite-element (FE) method. Gefen et al. ${ }^{12}$ performed a simulation on realistic two-dimensional alveolar geometries. The major advantage of this method is that it allows the investigation of the strain distribution within the alveolar wall, whereas the above-mentioned experimental methods only look at overall wall extension. Despite these advantages in this article they used a very simplified linear elastic constitutive model and a two-dimensional simulation for a three-dimensional problem. There are also several other groups investigating the mechanical behavior of artificially generated three-dimensional geometries. ${ }^{7,15,16}$ However, because of missing realistic images of alveolar geometries all these methods used symmetric octahedra and miss the complexity of the real geometry. In summary, none of the above-mentioned studies were able to determine the local threedimensional deformation state of the alveolar wall.

In this article we present a FE simulation of synchrotron-based X-ray tomographic microscopy (SRXTM) scanned alveolar geometries. This scanning method gives us, for the first time, high resolution three-dimensional images of alveoli located in the central regions of the lungs. Due to the high resolution we are able to model the alveolar walls in three dimensions, including the actual wall thickness. This allows us to gain a detailed insight into the behavior of single alveolar walls.

\section{MATERIALS AND METHODS}

To perform a FE analysis on SRXTM-based alveolar geometries several steps were necessary. First, precision-cut lung slices (PCLS) were prepared from isolated rat lungs as previously described. ${ }^{18,23}$ Second, the slices were scanned in the TOMCAT beamline of the Swiss Light Source (SLS). Third, a three-dimensional volume representation of the scanned images was created. Fourth, the three-dimensional volume was discretized with a volume mesh and boundary conditions were applied and finally, the problem was solved utilizing our advanced and well validated in-house research software platform BACI. ${ }^{31}$ In the following these steps are explained in more detail.

\section{Rat Lung Sample Preparation}

The samples have been prepared according to Schittny et al. ${ }^{23}$ The lungs of a rat at postnatal day 36 was intratracheally filled with $2.5 \%$ glutaraldehyde $\left(\mathrm{CH}_{2}\left(\mathrm{CH}_{2} \mathrm{CHO}\right)_{2}\right)$ in $0.03 \mathrm{M}$ potassium phosphate buffer ( $\mathrm{pH}$ 7.4) by instillation via tracheotomy at a constant pressure of $20 \mathrm{cmH}_{2} \mathrm{O}$. In order to prevent recoiling of the lung, we maintained this pressure during glutaraldehyde fixation. Subsequently, the lungs were dissected free and immersed in toto in the same fixative at a temperature of $4{ }^{\circ} \mathrm{C}$ for at least $24 \mathrm{~h}$. 
The samples were postfixed with $1 \%$ osmium tetroxide $\left(\mathrm{OsO}_{4}\right)$ and stained with $4 \%$ uranyl nitrate $\left(\mathrm{UO}_{2}\left(\mathrm{NO}_{3}\right)_{2}\right)$ to increase the X-ray absorption contrast. After dehydration in a graded series of ethanol we embedded the samples in Epon 812 using acetone as intermedium. The lung samples were glued onto small metal rods of $3.2 \mathrm{~mm}$ diameter using Araldite ${ }^{\mathrm{TM}}$.

The handling of animals before and during the experiments, as well as the experiments themselves, were approved and supervised by the local authorities.

\section{Beamline and Tomographic Imaging}

The experiments were performed at the TOMCAT beamline $^{25}$ at the SLS, Paul Scherrer Institut, Villigen, Switzerland. The samples were scanned at a beam energy of $12.601 \mathrm{keV}$, corresponding to a wavelength of $1 \AA$. After penetration of the sample, the X-rays were converted into visible light by a scintillator, magnified by diffraction limited microscope optics $(10 \times$ magnification) and digitized by a high-resolution $2048 \times$ 2048 pixel CCD camera (pco.2000, PCO AG, Kelheim, Germany) with 14 bit dynamic range. The detector was operated in $2 \times 2$ binning mode. As a result, each recorded projection had a size of $1024 \times 1024$ pixels with a size of $1.48 \mu \mathrm{m}$, the exposure time was $108 \mathrm{~ms}$.

We recorded 1501 projections at equiangular positions between 0 and $180^{\circ} \mathrm{C}$. The projections were postprocessed and rearranged into flat field-corrected sinograms prior to being reconstructed into tomographic slices on a 16-node computing cluster using a highly optimized filtered back-projection routine. Details of the imaging workflow and reconstruction setup have been described by Hintermüller et al. ${ }^{13}$ The resulting tomographic data set was a stack of 102416 bit tiff images, see Fig. 1.

\section{Segmentation}

In the next step the images were segmented utilizing the commercially available software Amira 4.1.2 (Mercury Computer Systems). For the segmentation itself we found the 'magic wand' tool, which is a combination of a threshold and region growth, to be the most efficient. In order to assess the accuracy of the segmented geometry, we compared the air-tissue ratio with previously published data. ${ }^{29}$ Our ratio showed good agreement (data not shown).

\section{Meshing and Boundary Conditions}

After creating the geometry a mesh was generated with the STL meshing package Harpoon (Sharc). Due to the complexity of the geometry, a new uniform nodal strain tetrahedral element with isochoric stabilization has been developed and used for the calculations, see Fig. 2. The element is based on linear interpolation of a classical displacement-based tetrahedral element formulation but applies nodal averaging of the deformation gradient to improve mechanical behavior, especially in the regime of near-incompressibility where classical linear tetrahedral elements perform very poorly. ${ }^{11}$

In a refinement study, we compared four different meshes, a summary of mesh details is given in Table 1. The base level represents the average size of one side of a regular tetrahedron. Example slices through the meshes with base levels of 4.23 and $2.11 \mu \mathrm{m}$ are shown in Fig. 2. The study showed that with a base level of $3.17 \mu \mathrm{m}$ the solution was mesh converged (data not shown).

To evaluate the influence of boundary effects, two different cubes, one with $158.57 \mu \mathrm{m}$ and the other one
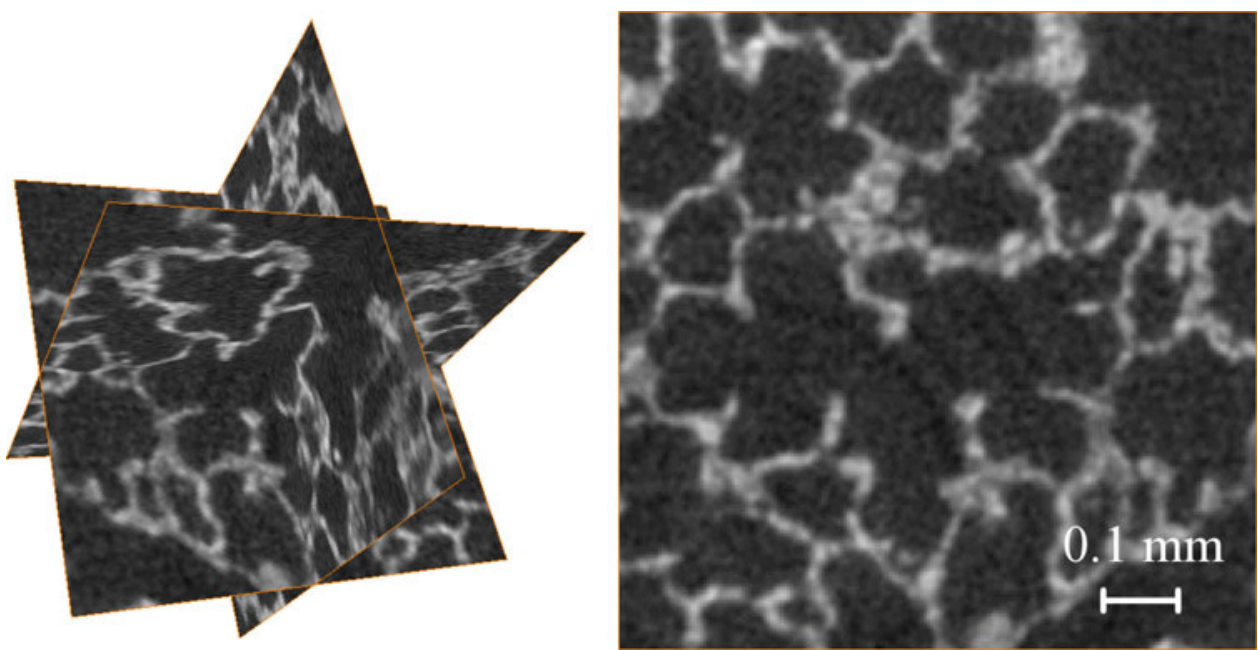

FIGURE 1. SRXTM image of rat lung parenchyma. The specimen was imaged in the TOMCAT beamline of the Swiss Light Source with a voxel size of $1.48 \times 1.48 \times 1.48 \mu \mathrm{m}$. 
(a)

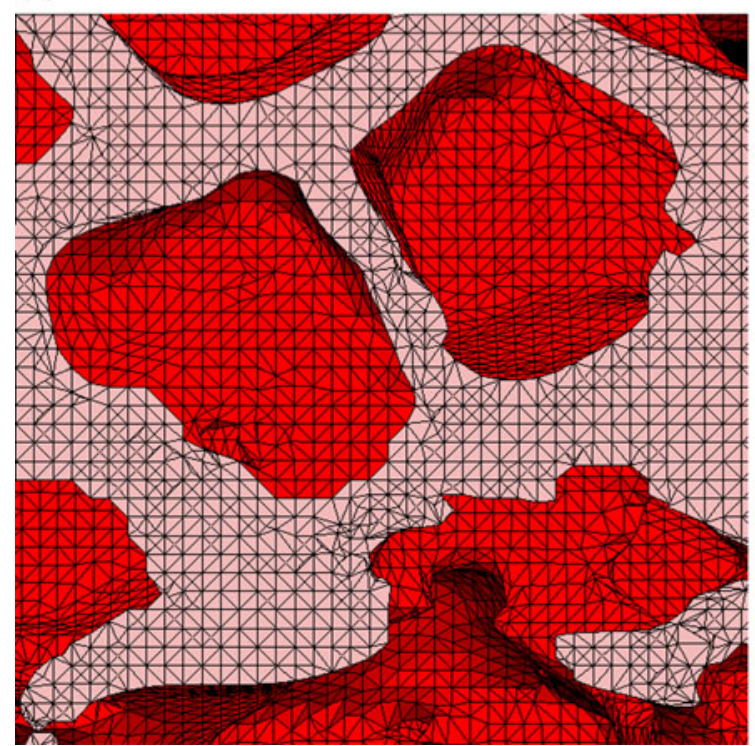

(b)

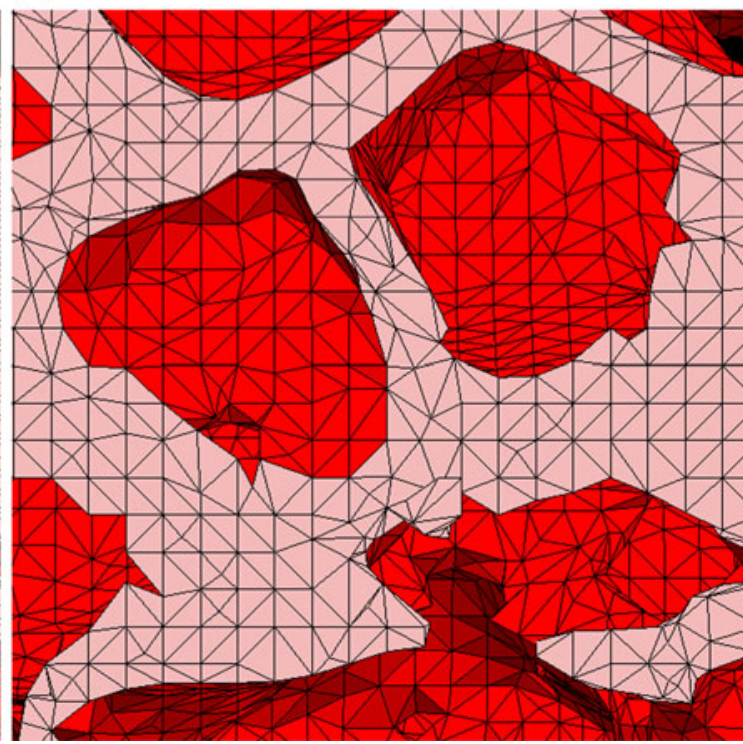

FIGURE 2. Cut through the mesh (a) with base level $2.11 \mu \mathrm{m}$ and (b) with base level $4.23 \mu \mathrm{m}$.

TABLE 1. Alveolar meshing statistics.

\begin{tabular}{lrc}
\hline Base level $(\mu \mathrm{m})$ & Elements & Degrees of freedom \\
\hline 5.29 & 577990 & 397020 \\
4.23 & 1164023 & 750297 \\
3.17 & 2727686 & 1669446 \\
2.11 & 8573680 & 5003121 \\
\hline
\end{tabular}

with $317.14 \mu \mathrm{m}$ side length, were compared, see Fig. 3. These cubes will be referred to as the small and the large cubes, respectively.

For our simulations we applied uniaxial tension and shear deformation to the samples. In both cases, the deformation was applied as a Dirichlet boundary condition. While the deformation of the bottom

small cube

large cube

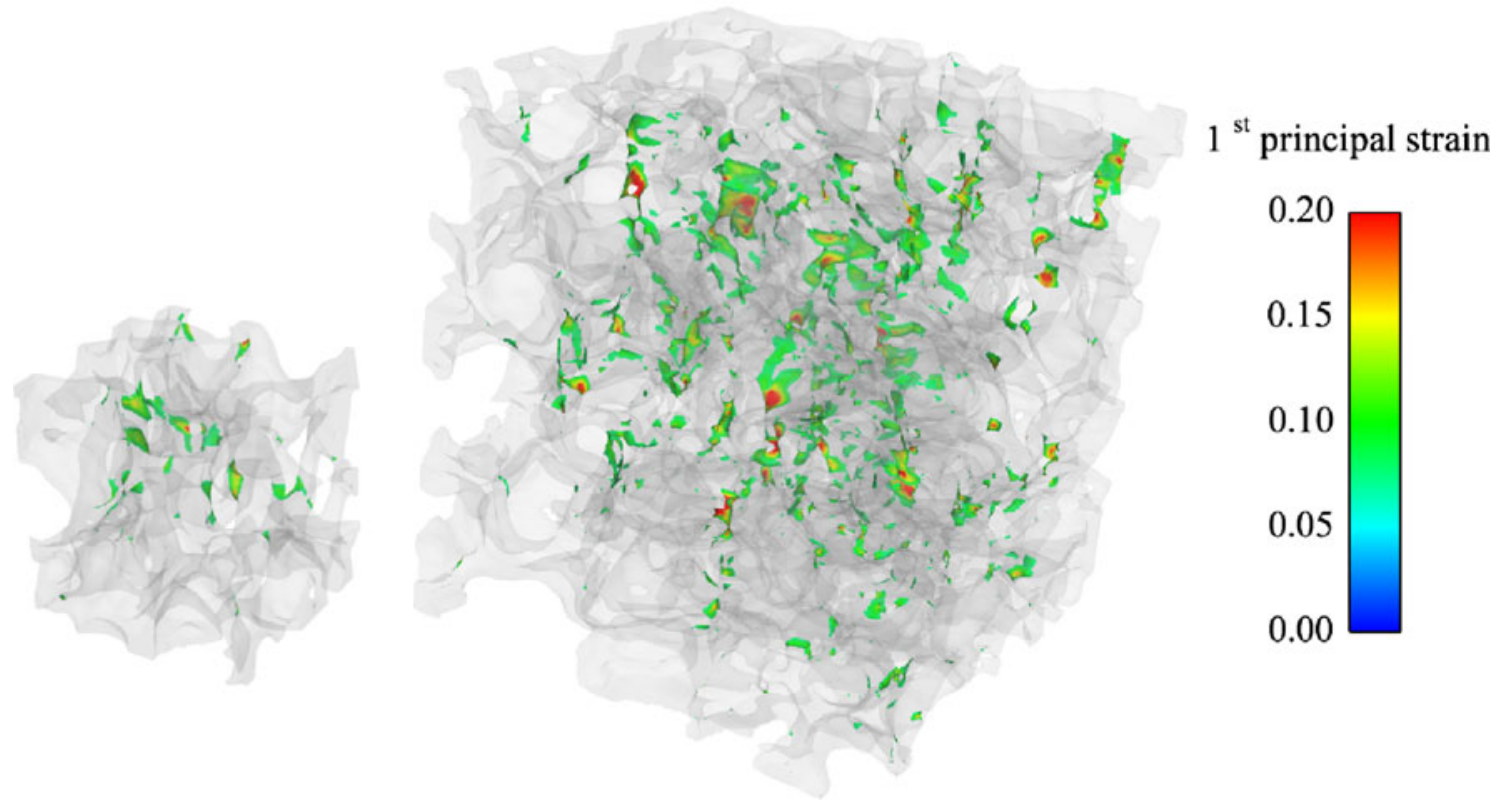

FIGURE 3. Location of 1st principal strain hotspots. Due to geometric complexity only strains larger than $10 \%$ are highlighted for $5 \%$ uniaxial elongation. 


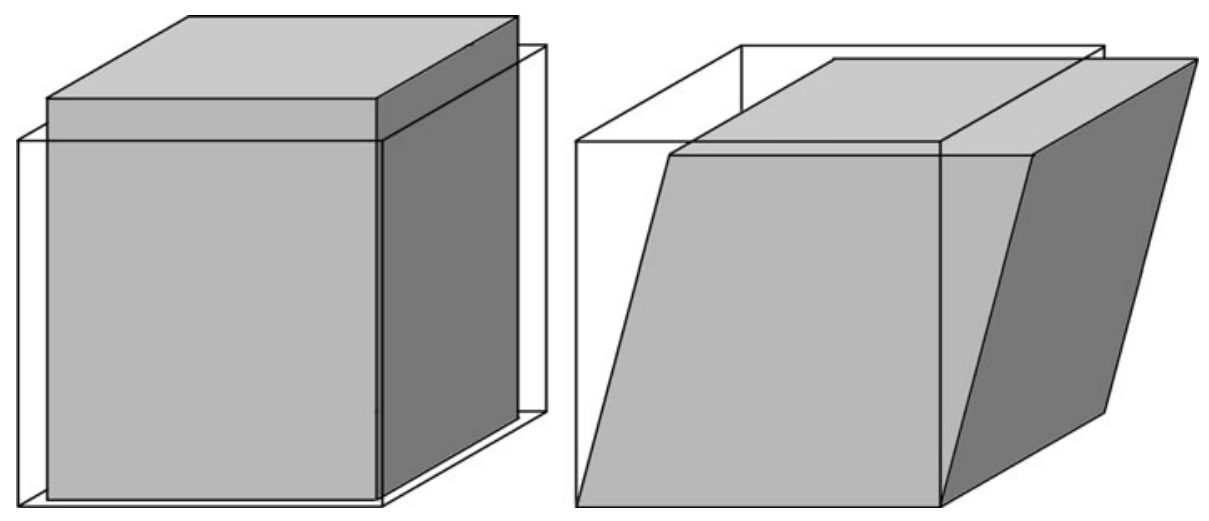

FIGURE 4. The tested deformation states are uniaxial tension and simple shear deformation.

surface was completely constrained, the top surface was either pulled upwards or pushed in the transverse direction, representing uniaxial tension and simple shear, respectively, see Fig. 4.

\section{Simulation}

The computation was performed with our advanced and well validated in-house multiscale and multiphysics research software platform BACI. ${ }^{31}$ The governing equation for the nonlinear mechanical behavior of the alveolar tissue is

$$
\rho \frac{D^{2} \mathbf{x}}{D t^{2}}=\nabla \cdot(\mathbf{F} \cdot \mathbf{S})+\rho \mathbf{b}
$$

in which $\rho$ is the density, $\mathbf{x}$ are the current positions, $\mathbf{F}$ is the deformation gradient tensor, which itself is defined as $\mathbf{F}=\frac{\partial \mathbf{x}}{\partial \mathbf{x}_{0}}$ with $\mathbf{x}_{0}$ denoting the reference position, $\mathbf{S}$ is the second Piola-Kirchhoff stress tensor, and $\mathbf{b}$ is the body force.

For the hyperelastic material model we postulate a strain energy density function (SEF)

$$
W:=W(\mathbf{C}),
$$

with $\mathbf{C}$ being the right Cauchy-Green deformation tensor, given by

$$
\mathbf{C}=\mathbf{F}^{T} \cdot \mathbf{F} .
$$

We obtain the corresponding second Piola-Kirchhoff stress tensor (S) through the first derivative of $W(\mathbf{C})$ with respect to $\mathbf{C}$

$$
\mathbf{S}=2 \frac{\partial W(\mathbf{C})}{\partial \mathbf{C}} .
$$

\section{Tissue and Material Model}

The alveolar tissue is represented by the walls between the airspace (alveolar septa). These walls are covered on both sides by an epithelial monolayer of alveolar type I and type II epithelial cells. The core of the walls contains a network of collagen fibrils and elastin fibers. In addition, it contains a sheet like network of capillaries. ${ }^{22}$ It was shown by Yuan et al. ${ }^{36,37}$ that collagen and elastin dominate the macroscopic elastic and dissipative properties of alveolar tissue, whereas the contribution of interstitial cells seems marginal. Previous studies ${ }^{24,28}$ suggest that the orientation of the fibers in the alveolar tissue is isotropic. This means we can formulate a hyperelastic SEF based only on invariants,

$$
\Psi(\mathbf{C})=\Psi\left(I_{1}, I_{3}\right) .
$$

where $I_{1}$ and $I_{3}$ are the first and the third invariant of the right Cauchy-Green deformation tensor, respectively, defined as

$$
I_{1}:=\operatorname{tr} \mathbf{C}, \quad I_{3}:=\operatorname{det} \mathbf{C} .
$$

In our simulations we are utilizing a Neo-Hookean formulation $^{14}$

$$
\Psi(\mathbf{C})=\frac{E(1-2 v)}{4 v+4 v^{2}}\left(I_{3}^{-\frac{v}{1-2 v}}-1\right)+\frac{E}{4-4 v}\left(I_{1}-3\right)
$$

with $E$ and $v$ being a Young's modulus like parameter and the Poisson's ratio, respectively. Due to the fact that the tissue mainly consists of water, it is considered as nearly incompressible $(v=0.49)$. The value for $E$ was fitted to experimentally determined curves $(E \approx 6.75 \mathrm{kPa})$, details of the experimental protocol can be found in Rausch et al. ${ }^{21}$

It is worthy to note that the SEF fulfills the principles of objectivity, material symmetry and also the requirements of polyconvexity and a stress-free reference state. Additionally, we make the assumption that due to the negative hydrostatic pressure in the pleural space the tissue is under tension all the time. ${ }^{26}$ This means tissue folding and unfolding at lower volumes is not considered. 


\section{RESULTS}

In a first step we identified strain hotspots meaning the regions of the tissue cube, with the highest strain values. Figure 3 shows a comparison of the strain hotspots for both cube sizes under 5\% uniaxial elongation. Due to the complex geometry only the 1 st (largest) strain eigenvalues larger than 0.1 are shown, the remaining tissue is set to be transparent.

The most important finding was that local strains are much higher than the global extension of the cubes. This was of course expected but now is quantified for the first time. It turned out that the local strains are up to four times higher than the global strains. Additionally, we found the strain hotspots to occur within the thinnest parts of the cube. This seems feasible since there is less tissue to resist the deformation. This leads to an uneven strain distribution throughout the parenchymal tissue. Thin regions become overstretched, whereas regions with tissue accumulation remain unchallenged. We further observed higher peak strains in the larger cube than in the smaller cube. This was potentially due to boundary effects (see later). This hypothesis is supported by the fact that in both cubes the hotspots occurred predominantly in the central regions.

In a next step a local hotspot was investigated in more detail. Figure 5 shows the distributions of the 1st, 2nd, and 3rd strain eigenvalues. The 1st eigenvalues are positive, whereas the $2 \mathrm{nd}$ and the 3 rd eigenvalues are both negative and of a much smaller magnitude. However, the strain patterns are similar for all three eigenvalues, i.e., the peak values occur in the thinnest part of the structure.

The corresponding eigenvectors, for a slice through this hotspot show the 1st eigenvectors pointing towards the pulling direction, whereas the 2nd and $3 \mathrm{rd}$ eigenvectors lie within the normal plane of the pulling direction (data not shown). It is also noteworthy that within the plane the eigenvectors do not follow a preferred direction. This behavior can be explained by the incompressibility of the tissue, i.e., if the tissue is stretched in one direction it has to be compressed in another direction. The compression seems to be quite evenly distributed within the normal plane of the pulling direction, therefore we do not see any preferred direction within this plane.

We also compared uniaxial tension with simple shear deformation, see Fig. 4. In both cases we have a deformation of $5 \%$ of the initial side length of the cube in the transversal direction for the shear deformation and in axial direction for the tensile deformation, see Fig. 6 .

Clearly the peak strain values are much higher for the uniaxial tension than for the shear deformation. However, they occur in similar regions within the geometry, i.e., the above-mentioned thinner parts of the structure. These observations are valid for all three strain eigenvalues. Additionally, we investigated the distribution of the eigenvectors in a strain hotspot (data not shown) and found the same distribution for the shear as for the tensile displacement, albeit of a differing magnitude.

Finally, to evaluate the influence of the boundary conditions we compared the strain distributions of four different cases. First, the small cube under 5\% uniaxial elongation (small cube), second, the large cube under $5 \%$ uniaxial elongation (large cube), third, the small cube under $5 \%$ shear deformation (shear) and fourth, the strain distribution within the center region of the large cube, i.e., the region of equivalent size to the small cube in the center of the large cube, which is challenged with $5 \%$ uniaxial elongation (center region), see Fig. 7. The boxes extend from the 25 th to the 75 th percentile, the red line in the middle indicates the median, and the whiskers range from the 0.01 to the $99.99 \%$ percentile. The additional dots within the boxes mark the location of the mean strain. It is obvious and expected that all distributions are skewed
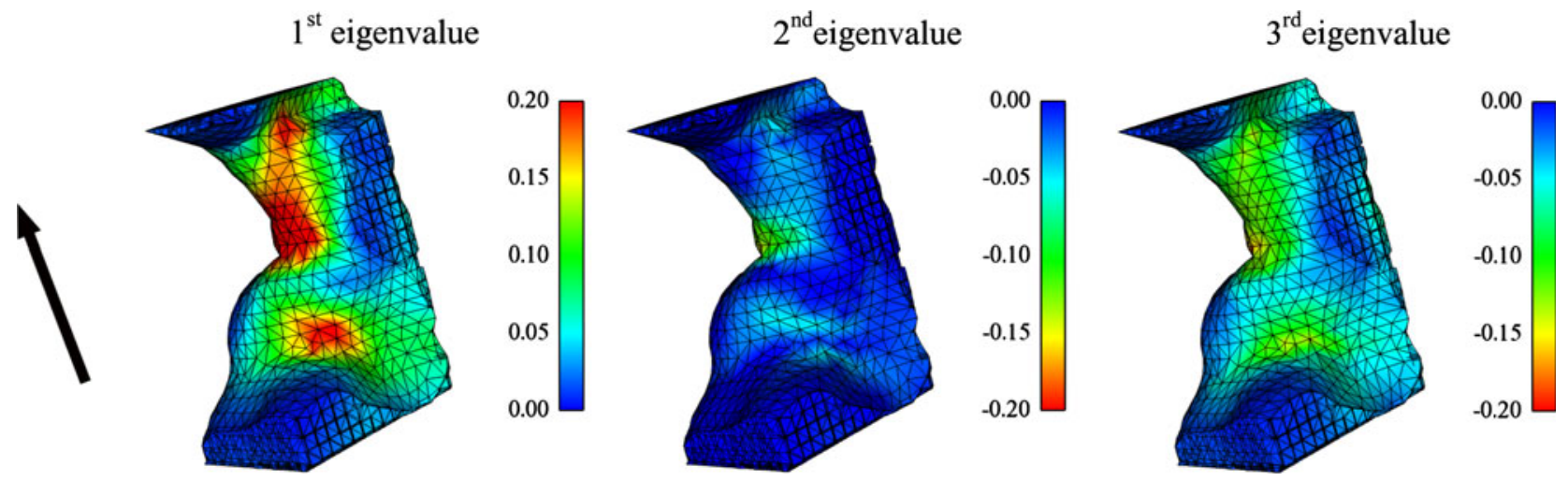

FIGURE 5. 1st, 2nd, and 3rd strain eigenvalues of a hotspot with the arrow indicating the direction of the uniaxial stretch. Due to better comparison the color maps of the 2 nd and 3rd strain eigenvalue are inverted. 

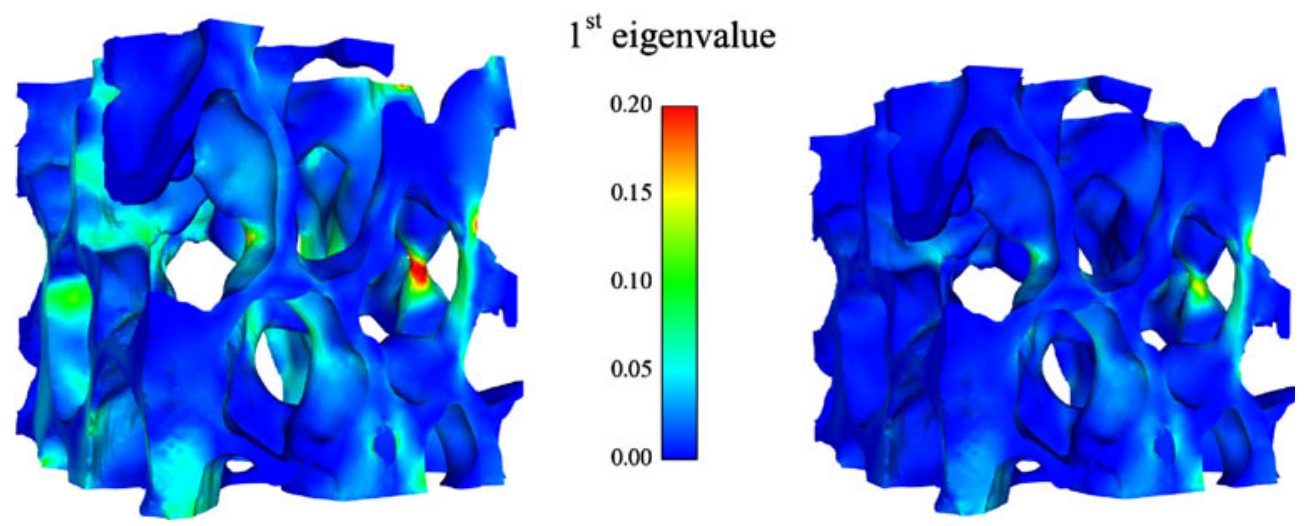

FIGURE 6. Comparison between uniaxial tension and shear deformation of the small cube (side length $158.57 \mu \mathrm{m}$ ). The colors indicate the first (largest) eigenvalue of the strain tensor.

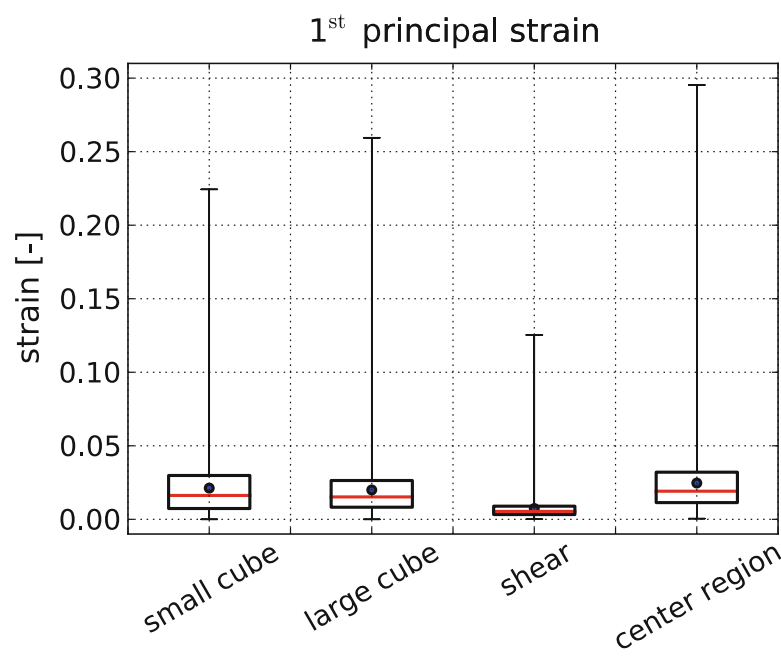

FIGURE 7. Comparison of the 1st principal strain distributions for four different cases. First, the small cube under $5 \%$ uniaxial elongation (small cube), second, the large cube under $5 \%$ uniaxial elongation (large cube), third, the small cube under $5 \%$ shear deformation (shear), and fourth, the strain distribution within the center region of the large cube, i.e., the region of equivalent size to the small cube in the center of the large cube, which is challenged with $5 \%$ uniaxial elongation (center region). The whiskers include $99.98 \%$ and the boxes $50 \%$ of all measurement points (outliers are not shown). The red lines in the center of the boxes are the medians and the dots are means.

towards lower strain values, since we have only a few strain hotspots. One of the main findings is that even though the mean and the standard deviation are higher for the small cube, the extreme values are higher for the large cube, see Table 2. When we consider only the central region of the large cube, the mean, standard deviation and median are higher than all other scenarios. Additionally, the difference between the mean and the median which can be seen as a measurement of the skewness of the distribution, is greatest. This backs up our assumption that more strain hotspots are developed in the larger cube, due to a reduction of
TABLE 2. Comparison of the 1st strain distributions.

\begin{tabular}{lcc}
\hline & Mean \pm sd & Median \\
\hline Small cube & $0.0212 \pm 0.0191$ & 0.0162 \\
Large cube & $0.0200 \pm 0.0178$ & 0.0152 \\
Shear & $0.0073 \pm 0.0074$ & 0.0054 \\
Center region & $0.0247 \pm 0.0199$ & 0.0191 \\
\hline
\end{tabular}

boundary effects. However, to put this in perspective it has to be mentioned that this mainly affects the outliers whereas the main distributions are rather similar.

Another interesting fact is that the mean values of all four evaluated distributions are at least twice as small as the $5 \%$ global strain. Furthermore, $90 \%$ of the local strains are below $5 \%$. This shows clearly that there are only certain hotspots in the tissue, which are overstrained, whereas the majority of the tissue remains within a healthy deformation state.

Finally, for the shear deformation, we found the distribution to have a much smaller mean and standard deviation. The smaller standard deviation was expected due to the more uniform deformation in the cube and the lower mean arose due to the smaller amount of hotspots.

\section{DISCUSSION}

In this article, we have presented FE simulations on SRXTM-based alveolar geometries. This method allowed us, for the first time, to determine local threedimensional strain states in real highly resolved alveolar geometries.

Comparing our method to previous experimental approaches, ${ }^{1,2,8,20}$ which can only calculate an averaged extension for each of the alveolar walls, our method is able to determine a three-dimensional strain state throughout the thickness of the tissue. 
A direct comparison with other numerical approaches is difficult, since the studies in literature are mainly investigating the effects of very specific components, $, 7,8,12,20$ rather than developing a general model. Additionally they are investigating these effects on very regular artificially generated geometries, which of course reduces the heterogeneity of the strain field. The only other FE study on real alveolar geometries was performed by Gefen et al. ${ }^{12}$ and is limited to two-dimensional geometries.

A further advantage of our method is the quality of our newly developed stabilized node-based uniform strain tetrahedron. This element allowed us to discretize our models with tetrahedral elements. The problem with normal tetrahedral elements is that they produce parasitic stresses for nearly incompressible materials (volumetric locking), leading to too stiff behavior. However, due to the complex geometry it is impossible to mesh the models with hexahedral elements.

From the simulation results we have two main conclusions; first there are certain hotspots in the alveolar geometry which are especially at risk for overstretching. These obviously tend to be at the thinnest regions. Second, a small global strain can lead to significant larger local strains, for uniaxial tension it can rise up to the fourfold. These conclusions were found to be independent of the loading type.

Looking at in vitro experiments on alveolar type II cells ${ }^{3,5,19,30}$ there is a wide diversity of how much stretch causes inflammatory reactions. The numbers range between strains of 0.05 up to strains of 0.3 . Comparing these values with the local peak strains found in our simulations we find a global strain of 0.05 would be sufficient to cause inflammation in all of those cases. This presents an interesting observation as it suggests that the amount of stretching done in these experiments may not be representative of the in vivo environment or at the very least maybe an underestimation. This large increase in strain from the global to local level shows that inflammatory reactions potentially initiate much earlier than previously thought.

For a further verification of the dependency on boundary effects, we want to include the surrounding alveolar tissue in our simulations. For this reason we are working on including the presented simulations within a multiscale approach for alveolar ensemble. ${ }^{33}$ This allows us to project the global parenchymal deformation down to the level of a single alveolar ensemble in order to provide realistic boundary conditions. This method has the advantage that we will be able to measure local alveolar strain fields in large geometries, for example living precision cut rat lung slices (PCLS). Dassow et al. ${ }^{6}$ recently measured calcium fluxes, which are known to be induced by ventilatory lung stretch, within the alveolar wall of these
PCLS using a bioreactor. With this experimental approach and our computational models we would be able to compare the local strain fields in PCLS directly with the locations of increased calcium fluxes, hence providing a mechanical-biological pathway for the initiation of ventilator-induced lung inflammation.

Furthermore, by combining an inverse analysis ${ }^{21}$ with this multiscale approach we want to determine a more sophisticated constitutive model for individual alveolar walls. This combined method will utilize the resolved real alveolar geometries embedded into experimentally tested specimens. Finally we will also combine our surfactant model $^{34}$ with these simulations of realistic alveolar geometries. Due to the fact that the presented model does not include any surface tension, we would expect an overall stiffer behavior after the inclusion of our surfactant model, even though the surfactant molecules reduce the surface tension. Performing a simple thought experiment where we simplify the regions of the strain hotspots as incompressible cylinders we can calculate that when the length of the cylinder increases by $10 \%$ the surface area of the cylinder increases by $4.88 \%$. This increase of the surface area leads to a counteracting force arising from the existing surface tension. Additionally, the deformation happens within a small time scale, which could lead to a temporal reduction of the concentration of surfactant molecules, leading to even higher surface tension in the regions of larger deformations.

In the future we also want to modify artificially generated alveolar geometries, so that they result in similar strain distributions as the real alveolar geometries. This has the advantage, that these models could much simpler and more efficiently be included in our overall lung model. ${ }^{32}$

With this model it will be possible to investigate how novel ventilation strategies, e.g., how variable tidal volume ventilation affect the deformations at the alveolar level. This will be done by first considering how the airflow distributes in the large airways, ${ }^{4}$ how this couples down to the more peripheral levels and then finally via the aforementioned multiscale approach the deformation in the alveolar wall. Understanding the influence of such ventilation strategies on local strain in the individual alveolar walls is of central importance as it indicates, by implication, locations where the onset of inflammation may occur.

\section{ACKNOWLEDGMENTS}

Support by the German Science Foundation/Deutsche Forschungsgemeinschaft DFG and the TUM Graduate School is gratefully acknowledged. We also gratefully acknowledge the help of Christian Martin and Stefan Uhlig for providing us with the PCLSs. 


\section{REFERENCES}

${ }^{1}$ Brewer, K., H. Sakai, A. M. Alencar, A. Majumdar, S. P. Arold, K. R. Lutchen, E. P. Ingenito, and B. Suki. Lung and alveolar wall elastic and hysteretic behavior in rats: effects of in vivo elastase treatment. J. Appl. Physiol. 95(5):1926-1936, 2003.

${ }^{2}$ Cavalcante, F. S., S. Ito, H. Sakai, A. M. Alencar, M. P. Almeida, I. S. Andrade, A. Majumdar, E. P. Ingenito, and B. Suki. Mechanical interactions between collagen and proteoglycans: implications for the stability of lung tissue. J. Appl. Physiol. 98(2):672-679, 2005.

${ }^{3}$ Chandel, N. S., and J. I. Sznajder. Stretching the lung and programmed cell death. Am. J. Physiol. Lung Cell Mol. Physiol. 279(6):1003-1004, 2000.

${ }^{4}$ Comerford, A., C. Förster, and W. A. Wall. Structured tree impedance outflow boundary conditions for 3D lung simulations. J. Biomech. Eng. 132(8):081002, 2010.

${ }^{5}$ Copland, I. B., and M. Post. Stretch-activated signaling pathways responsible for early response gene expression in fetal lung epithelial cells. J. Cell. Physiol. 210(1):133-143, 2007. ${ }^{6}$ Dassow, C., L. Wiechert, C. Martin, S. Schumann, G. Müller-Newen, O. Pack, J. Guttmann, W. A. Wall, and S. Uhlig. Biaxial distension of precision-cut lung slices. $J$. Appl. Physiol. 108:713-721, 2010.

${ }^{7}$ Denny, E., and R. C. Schroter. A model of non-uniform lung parenchyma distortion. J. Biomech. 39(4):652-663, 2006.

${ }^{8}$ DiRocco, J. D., L. A. Pavone, D. E. Carney, Ch. J. Lutz, L. A. Gatto, S. K. Landas, and G. F. Nieman. Dynamic alveolar mechanics in four models of lung injury. Intensive Care Med 32(1):140-148, 2006.

${ }^{9}$ Dos Santos, C. C., and A. S. Slutsky. Invited review: mechanisms of ventilator-induced lung injury: a perspective. J. Appl. Physiol. 89(4):1645-1655, 2000.

${ }^{10}$ Dos Santos, C. C., and A. S. Slutsky. The contribution of biophysical lung injury to the development of biotrauma. Annu. Rev. Physiol. 68:585-618, 2006.

${ }^{11}$ Gee, M. W., C. R. Dohrmann, S. W. Key, and W. A. Wall. A uniform nodal strain tetrahedron with isochoric stabilization. Int. J. Numer. Methods Eng. 78(4):429-443, 2009.

${ }^{12}$ Gefen, A., D. Elad, and R. J. Shiner. Analysis of stress distribution in the alveolar septa of normal and simulated emphysematic lungs. J. Biomech. 32(9):891-897, 1999.

${ }^{13}$ Hintermüller, C., F. Marone, A. Isenegger, and M. Stampanoni. Image processing pipeline for synchrotronradiation-based tomographic microscopy. J. Synchrotron Radiat. 17(4):550-559, 2010.

${ }^{14}$ Holzapfel, G. A. Nonlinear Solid Mechanics: A Continuum Approach for Engineering. New York: Wiley, 2001.

${ }^{15}$ Karakaplan, A. D., M. P. Bieniek, and R. Skalak. A mathematical model of lung parenchyma. J. Biomech. Eng. 102(2):124-136, 1980.

${ }^{16}$ Kowe, R., R. C. Schroter, F. L. Matthews, and D. Hitchings. Analysis of elastic and surface tension effects in the lung alveolus using finite element methods. J. Biomech. 19(7): 541-549, 1986.

${ }^{17}$ Maksym, G. N., J. J. Fredberg, and J. H. T. Bates. Force heterogeneity in a two-dimensional network model of lung tissue elasticity. J. Appl. Physiol. 85:1223-1229, 1998.

${ }^{18}$ Martin, C., S. Uhlig, and V. Ullrich. Videomicroscopy of methacholine-induced contraction of individual airways in precision-cut lung slices. Eur. Respir. J. 9(12):2479-2487, 1996.

${ }^{19}$ Ning, Q., and X. Wang. Response of alveolar type ii epithelial cells to mechanical stretch and lipopolysaccharide. Respiration 74(5):579-585, 2007.
${ }^{20}$ Perlman, C. E., and J. Bhattacharya. Alveolar expansion imaged by optical sectioning microscopy. J. Appl. Physiol. 103:1037-1044, 2007.

${ }^{21}$ Rausch, S. M. K., C. Martin, P. B. Bornemann, S. Uhlig, and W. A. Wall. Material model of lung parenchyma based on living precision-cut lung slice testing. J. Mech. Behav. Biomed. 4:583-592, 2011.

${ }^{22}$ Schittny, J. C., and P. H. Burri. Development and Growth of the Lung. Fishman's Pulmonary Diseases and Disorders. New-York: McGraw-Hill, 2008.

${ }^{23}$ Schittny, J. C., S. I. Mund, and M. Stampanoni. Evidence and structural mechanism for late lung alveolarization. Am. J. Physiol. Lung Cell Mol. Physiol. 294(2):246-254, 2008.

${ }^{24}$ Sobin, S. S., Y. C. Fung, and H. M. Tremer. Collagen and elastin fibers in human pulmonary alveolar walls. J. Appl. Physiol. 64(4):1659-1675, 1988.

${ }^{25}$ Stampanoni, M., A. Groso, A. Isenegger, G. Mikuljan, Q. Chen, A. Bertrand, S. Henein, R. Betemps, U. Frommherz, P. Böhler, D. Meister, M. Lange, and R. Abela. Trends in synchrotron-based tomographic imaging: the SLS experience. In: Society of Photo-Optical Instrumentation Engineers (SPIE) Conference Series, 2006.

${ }^{26}$ Suki, B., and J. H. T. Bates. Extracellular matrix mechanics in lung parenchymal diseases. Respir. Physiol. Neurobiol. 163:33-43, 2008

${ }^{27}$ The Acute Respiratory Distress Syndrome Network. Ventilation with lower tidal volumes as compared with traditional tidal volumes for acute lung injury and the acute respiratory distress syndrome. N. Engl. J. Med. 342(18): 1301-1308, 2000.

${ }^{28}$ Toshima, M., Y. Ohtani, and O. Ohtani. Three-dimensional architecture of elastin and collagen fiber networks in the human and rat lung. Arch. Histol. Cytol. 67(1):31-40, 2004.

${ }^{29}$ Tschanz, S. A., A. N. Makanya, B. Haenni, and P. H. Burri Effects of neonatal high-dose short-term glucocorticoid treatment on the lung: a morphologic and morphometric study in the rat. Pediatr. Res. 53(1):72-80, 2003.

${ }^{30}$ Vlahakis, N. E., M. A. Schroeder, A. H. Limper, and R. D. Hubmayr. Stretch induces cytokine release by alveolar epithelial cells in vitro. Am. J. Physiol. 277(1):167-173, 1999.

${ }^{31}$ Wall, W. A., and M. Gee. Baci: A Parallel Multiphysics Simulation Environment. Technical Report, Institute for Computational Mechanics, TUM, 2010.

${ }^{32}$ Wall, W. A., L. Wiechert, A. Comerford, and S. Rausch. Towards a comprehensive computational model for the respiratory system. Int. J. Numer. Methods Biomed. Eng. 26(7):807-827, 2010.

${ }^{33}$ Wiechert, L., and W. A. Wall. A nested dynamic multiscale approach for 3D problems accounting for micro-scale multi-physics. Comput. Methods Appl. Mech. Eng. 199(2122):1342-1351, 2010.

${ }^{34}$ Wiechert, L., R. Metzke, and W. A. Wall. Modeling the mechanical behavior of lung tissue at the micro-level. $J$. Eng. Mech. 135(5):434-438, 2009.

${ }^{35}$ Wilson, T. A., and H. Bachofen. A model for mechanical structure of the alveolar duct. J. Appl. Physiol. 52:10641070, 1982.

${ }^{36}$ Yuan, H., E. P. Ingenito, and B. Suki. Dynamic properties of lung parenchyma: mechanical contributions of fiber network and interstitial cells. J. Appl. Physiol. 83(5):14201431, 1997 (discussion 1418-9).

${ }^{37}$ Yuan, H., S. Kononov, F. S. Cavalcante, K. R. Lutchen, E. P. Ingenito, and B. Suki. Effects of collagenase and elastase on the mechanical properties of lung tissue strips. J. Appl. Physiol. 89(1):3-14, 2000. 